\title{
FORMULATION AND EVALUATION OF AMPHOTERICIN B AND MILTEFOSINE COMBINATION NANOVESICLES
}

\author{
MULUGETA F. BEZABEH ${ }^{1}$, KARL A. WERBOVETZ ${ }^{2}$, K. V. RAMANA MURTHY ${ }^{1}$
}

${ }^{1}$ College of Pharmaceutical Sciences, Andhra University, Visakhapatnam 530003, India, ${ }^{2}$ Division of Medicinal Chemistry and Pharmacognosy, College of Pharmacy, the Ohio State University 43210, USA

Email: befentie@gmail.com

Received: 23 Dec 2020, Revised and Accepted: 15 Feb 2021

\section{ABSTRACT}

Objective: The aim of the present investigation is to formulate and evaluate amphotericin B-miltefosine combination nanovesicles for application in the treatment of visceral leishmaniasis.

Methods: Amphotericin B-miltefosine combination (AmB-MTF) nanovesicles were prepared by ethanol injection method. Formulations of nanovesicles were evaluated at varying conditions of lipids composition, drug-lipid proportion, ethanol-water composition and stirring rate, on drug entrapment efficiency and particle size.

Results: The study showed that entrapment efficiency was significantly affected $(\mathrm{p}<0.01)$ by the effects of lipids composition, drug-lipid proportion, ethanol-water composition, and stirring rate. Particle size of nanovesicles was significantly affected $(p<0.05)$ by drug-lipid proportion and stirring rate. An optimized formulation of amphotericin B-miltefosine nanovesicles was prepared at optimal factors composition of: phosphatidylcholinecholesterol-stearic acid 20:4:1, drug-lipid 1:8, AmB-MTF 1:1; ethanol-water 1:4 ratios, and stirring rate 1000 rpm. The AmB-MTF 1:1 nanovesicles formulation showed particle size of $145.6 \mathrm{~nm}$, poly dispersity index 0.19 , zeta potential- $27.3 \mathrm{mV}$ and drug entrapment efficiency $87 \%$.

Conclusion: Evaluation of AmB-MTF 1:1 nanovesicles showed development of a successful formulation with very good compatibility, extended drug release, convenient vesicle size and high drug entrapment efficiency. To conclude, AmB-MTF 1:1 nanovesicles formulation could be a safe and reliable therapeutic option over the conventional combination therapy provided further antileishmanial investigations are investigated in vitro and in vivo.

Keywords: Nanovesicles, AmB-MTF, Ethanol injection method, Leishmaniasis

(C) 2021 The Authors. Published by Innovare Academic Sciences Pvt Ltd. This is an open access article under the CC BY license (https://creativecommons.org/licenses/by/4.0/) DOI: https://dx.doi.org/10.22159/ijap.2021v13i3.40605. Journal homepage: https://innovareacademics.in/journals/index.php/ijap

\section{INTRODUCTION}

Visceral leishmaniasis is the most severe form of leishmaniasis by which patients nearly always die if untreated. It causes half a million new cases and 50,000 deaths each year. Visceral leishmaniasis remains to be a challenge for poor people in developing countries. The current treatment for visceral leishmaniasis is with drugs developed 50 y ago. Pharmaceutical companies are not attracted for developing new therapeutic options for neglected infectious diseases due to low incentives in the area [1,2].

Hence, the best strategy that remains as effective solution in crisis associated with the neglected diseases is using combination of the exciting drugs and developing novel drug delivery systems to improve the efficacy, specificity, tolerability, and emergence of resistance of existing antiparasitic agents [3]. Nanocarriers drug delivery to the liver and spleen, the main infection site of visceral leishmaniasis, have been developed [4]. Ambisome is the most effective and safe amphotericin B lipid liposomal formulation currently available on market. However, ambisome monotherapy unresponsiveness and drug resistance cases have also been reported in some places such as Bahir, India [5].

In combating the risk of resistance and to increase the efficacy of the existing drugs, WHO recommended use of combination of drugs. WHO recommended the use of liposomal AmB ( $5 \mathrm{mg} / \mathrm{kg}$ by infusion, single dose) and miltefosine (daily for $7 \mathrm{~d}$ orally) for the treatment of visceral leishmaniasis caused by $L$. donovani in Bangladesh, Bhutan, India and Nepal. This decreases the treatment duration by monotherapy from $28 \mathrm{~d}$ of miltefosine and 6-10 d of liposomal AmB to only $7 \mathrm{~d}$ combination therapy at reduced total exposure of each drug to patients $[1,6]$. However, the two drugs are given in different routes and as separate dosage forms, and miltefosine is given in its conventional delivery that has significant gastrointestinal disturbances and hemolytic effects. Thus, the objective of the present investigation is to formulate and evaluate AmB-MTF combination lipid nanovesicles in a single dosage form and for targeted drug delivery to infection sites in the treatment of visceral leishmaniasis.

\section{MATERIALS AND METHODS}

\section{Materials}

Amphotericin B (Sigma St Louis, USA), miltefosine (Cayman chemicals, USA), phosphatidylcholine (Spectrum chemicals, USA), cholesterol (MB Biomedicals, USA), oleic acid (Fisher; USA), lecithin (Fisher; USA) and stearic acid (Fisher; USA) were purchased. Distilled water, tween 80 (Fisher chemicals; USA), methanol (Fisher chemicals; USA), ethyl alcohol (Dacon Laboratories, USA), dimethyl sulfoxide (Fisher chemicals, USA), phosphate buffered saline tablets (Fisher chemicals, USA), and all other reagents were all used as received. All chemicals used were analytical grade.

\section{Methods}

\section{Preparation of nanovesicles}

Drug loaded nanovesicles were prepared using ethanol injection technique based on Tanga et al. [7]. Accurately weighed AmB was dissolved in dimethyl sulfoxide (DMSO) to form $25 \mathrm{mg} / \mathrm{ml}$ solution. Specified amounts of lipids and $5 \mathrm{mg}$ of miltefosine were accurately measured and dissolved in ethanol and heated to $75{ }^{\circ} \mathrm{C}$ as per the compositions given in table 1 . A required amount of $\mathrm{AmB}$ solution was preheated to $75^{\circ} \mathrm{C}$ and added to the lipid solution. The ethanolic solution was kept in sonicator bath for about $10 \mathrm{~min}$ to dissolve the entire content in ethanol with the end point as a clear yellowish solution. This solution was heated to $75^{\circ} \mathrm{C}$ in the hot plate water bath (Heidolph magnetic stirrers 0416, Germany), injected into phosphate buffer saline (PBS) (pH 7.4) aqueous medium preheated at $75{ }^{\circ} \mathrm{C}$ in a round bottom flask, stirred continuously at a required rate for $5 \mathrm{~min}$ and probe sonicated (Microson TM, XL2000, USA) for 1 minute at a frequency of $20 \mathrm{kHz}$. The hot water bath was removed and the magnetic stirring was further continued at the same speed for about $15 \mathrm{~min}$ to bring the system to room temperature. Ethanol 
was removed from the preparation by using rotavapor (Heidolph Bushi Rotavapor, R-114, Germany). Finally, the vesicular dispersion was stored in airtight amber glass container at $2-8^{\circ} \mathrm{C}$.

\section{Drug entrapment efficiency}

Drug entrapment efficiency (DEE) was estimated according to Lankallapalli et al., and Bose et al., [8,9]. Nanovesicles suspension containing an equivalent of $1 \mathrm{mg} \mathrm{AmB}$ was diluted 1 to 5 with DMSOdistilled water $(1: 25 \mathrm{v} / \mathrm{v})$ and centrifuged (Eppendruf AG 5404, Germany) at $20,000 \mathrm{~g}$ for $30 \mathrm{~min}$ at $4{ }^{\circ} \mathrm{C}$. The supernatant was carefully separated and kept in a separate glass tube. For entrapped drug, the precipitate was dissolved in $5 \mathrm{ml}$ DMSO-methanol $(1: 5 \mathrm{v} / \mathrm{v}) .1 \mathrm{ml}$ of this solution was diluted appropriately to make a solution of $20 \mathrm{ml}$ in DMSO-methanol-water (1:4:5 v/v) and AmB was determined directly from this solution at $408 \mathrm{~nm}$ using UV-Vis spectroscopy (Hitachi-2910, Japan). For the unentrapped drug, $1 \mathrm{ml}$ of the clear supernatant solution collected previously was diluted appropriately to form $5 \mathrm{ml}$ solution in DMSO-methanol-water (1:4:5) and AmB was determined directly from this solution at $408 \mathrm{~nm}$. The total drug content was obtained as sum of drug content in the supernatant and in the precipitate. The AmB entrapment efficiency was calculated by using the following formula. In the case of miltefosine, entrapment efficiency is considered to be $100 \%$, because it is the structural part of the nanovesicles due to its surfactant like action [10].

$$
\text { DEE }(\%)=\frac{\text { Entraped drug content }}{\text { Total drug content }} \times 100
$$

\section{Particle size and zeta potential determination}

The average particle size, polydispersity index (PDI) and zeta potential measurements were evaluated by dynamic light scattering technique. All analyses were carried out using Zeta sizer (Zita sizer, USA) for nanoparticles. The measurements of particle size, polydispersity index and zeta potential for each sample were carried out in triplicates and reported as mean values [11].

\section{In vitro drug release studies}

In vitro release of $\mathrm{AmB}$ from nanovesicles was evaluated by the dialysis bag diffusion technique $[9,12]$. Sample of nanovesicles suspension equivalent to $2 \mathrm{mg} \mathrm{AmB}$ was diluted to $5 \mathrm{ml}$ in PBS (pH 7.4). The resulting $5 \mathrm{ml}$ solution was transferred to dialysis tubing (MW cutt-off 14,000 Da, Ward's science, USA) which has been priorly soaked for $15 \mathrm{~min}$ in distilled water. The dialysis tubing with the $5 \mathrm{ml}$ sample in was sealed at both ends, and immersed into a receptor compartment containing $95 \mathrm{ml}$ dissolution medium of PBS
(pH 7.4) and 1\% tween 80. The receptor compartment was stirred at $100 \mathrm{rpm}$ and maintained at $37 \pm 0.5^{\circ} \mathrm{C}$. The receptor compartment was covered to prevent the evaporation of release medium. $2 \mathrm{ml}$ of samples were withdrawn at time intervals $(0,2,4,6,12,24,48$ and $72 \mathrm{~h}$ ), and the same volume was replaced by fresh medium at same temperature. The sample solutions in DMSO-methanol-water (1:4:5) were prepared using appropriate dilution and were analyzed by UVVis spectroscopy at $408 \mathrm{~nm}$. All the experiments were performed in triplicate, and the average values were taken.

\section{Fourier transform infrared spectroscopy}

The Fourier transform infrared (FTIR) spectra of AmB and miltefosine plain drugs, and freeze-dried AmB-MTF nanovesicle formulations were analyzed using a FTIR spectrophotometer (Thermo Nicholet 6700, Portugal) [11, 12]. Samples were diluted with $\mathrm{KBr}$ powder in a pellet die, and the mixture was pressed at high pressure gauge to form a thin transparent disc. The FTIR measurements were performed at wave numbers ranging from 4000 to $450 \mathrm{~cm}^{-1}$ at constant rate of $10^{\circ} \mathrm{C} /$ minute under an argon purge.

\section{RESULTS AND DISCUSSION}

AmB-MTF nanovesicles were prepared by ethanol injection method. Nanovesicles were evaluated at different conditions of lipids composition, drug-lipid proportion, ethanol-water composition and stirring rate on drug entrapment efficiency and particle size. In the preparation of nanovesicles, ethanol soluble components; phosphatidylcholine (PC), cholesterol, stearic acid and miltefosine were first dissolved in ethanol at $75{ }^{\circ} \mathrm{C}$ to assure a homogeneous mixture of lipids for spontaneous formation of small unilamellar vesicles (SUVs) upon injection [12]. AmB was dissolved in DMSO (25 $\mathrm{mg} / \mathrm{ml}$ ) to enhance its entrapment in nanovesicles. This is in accord with previous findings that the amount of poorly water soluble drugs encapsulated into vesicles is usually quite high with solvent aid [14]. Due to limited drug solubility in the external aqueous phase, the solubilized AmB in organic solvents tended to have a higher affinity to the phospholipid membrane components of the nanovesicles, thereby resulting in enhanced entrapment efficiency [14]. The concentration of lipids in ethanol solution was fixed at around $25 \mathrm{mg} / \mathrm{ml}$ to insure formation of SUVs in the vesicle formation. Studies showed that decreasing phospholipid concentrations in ethanol from $50 \mathrm{mg} / \mathrm{ml}$ to about $10 \mathrm{mg} / \mathrm{ml}$ produced SUVs of a significant lower particle size, although entrapment efficiency may be decreased as the lipid concentration decreased [15]. The effects of ingredients and process variables on nanovesicles formulations is presented in table 1 .

Table 1: Effects of different factors in preparation of AmB-MTF nanovesicles

\begin{tabular}{|c|c|c|c|}
\hline Factors & & Particle size (nm) & DEE (\%) \\
\hline \multirow[t]{4}{*}{ Stirring rate (rpm) } & $8 \mathrm{mg}$ cholesterol, $30 \mathrm{mg}$ PC, 1:10 AmB: lipid and 1:4 Ethanol: $P B S$. & & \\
\hline & 500 & $196.3 \pm 6.9$ & $81.45 \pm 2.26$ \\
\hline & 1000 & $193.6 \pm 6.3$ & $79.05 \pm 5.41$ \\
\hline & 1200 & $167.6 \pm 14.6$ & $67.97 \pm 2.96$ \\
\hline \multirow[t]{5}{*}{ Lipid (mg) } & 1000 rpm,1:10 AmB: lipid, and 1:4 Ethanol: $\mathrm{PBS}$, total lipid (40 mg) & & \\
\hline & Stearic acid, Oleic acid, Tween 80 (10:1:1) & $171.5 \pm 10.7$ & $37.41 \pm 4.8$ \\
\hline & Stearic acid,Lecithin, Cholesterol (0.5:7:2) & $184.0 \pm 11.7$ & $45.43 \pm 5.77$ \\
\hline & PC, Cholesterol (7:2) & $192.5 \pm 9.0$ & $77.63 \pm 2.73$ \\
\hline & Stearic acid, PC, Cholesterol (0.5:7:2) & $193.3 \pm 6.9$ & $93.07 \pm 3.19$ \\
\hline \multirow[t]{4}{*}{ Drug: lipid (mg) } & $1000 \mathrm{rpm}, 8 \mathrm{mg}$ cholesterol, $30 \mathrm{mg} \mathrm{PC}$, and 1:4 Ethanol: PBS. & & \\
\hline & $1: 5$ & $202.6 \pm 1.7$ & $61.26 \pm 4.39$ \\
\hline & 1:10 & $192.5 \pm 6.3$ & $79.43 \pm 5.49$ \\
\hline & $1: 20$ & $169.7 \pm 17.4$ & $88.54 \pm 3.31$ \\
\hline \multirow[t]{4}{*}{ Ethanol-PBS(pH 7.4) } & $1000 \mathrm{rpm}, 8 \mathrm{mg}$ cholesterol, $30 \mathrm{mg}$ PC, 1:10 AmB: lipid & & \\
\hline & $2: 1$ & --- & --- \\
\hline & $1: 1$ & $184.6 \pm 3.3$ & $38.34 \pm 4.09$ \\
\hline & $1: 4$ & $193.6 \pm 6.3$ & $79.43 \pm 5.49$ \\
\hline
\end{tabular}

Values represent mean $\pm \mathrm{SD}(\mathrm{n}=3)$, weight of miltefosine in all formulations was $5 \mathrm{mg}$, total ethanol-water volume was $10 \mathrm{ml}$.

\section{Effect of stirring rate}

The effect of stirring rate on vesicle size and entrapment efficiency was assessed and the results are presented in table 1 . In order to investigate the effect of stirring rate, formulations were prepared at
500,1000 and $1200 \mathrm{rpm}$ while all other factors put at specified values in the table. The results showed particle size decreased from 193.6 to $167.6 \mathrm{~nm}$ when the stirring rate was increased from 1000 to 1200 rpm. The particle size also decreased from 196.3 to $193.6 \mathrm{~nm}$ by increasing stirring rate from 500 to $1000 \mathrm{rpm}$. Overall, the results 
showed increasing stirring rate from 500 to $1200 \mathrm{rpm}$ significantly decreased the size of nanovesicles from 196.3 to $167.6 \mathrm{~nm}(\mathrm{p}<0.05)$. This may be due to the increased shearing action that has decreased aggregation of small vesicles leading to formation of smaller particle size vesicles [16]. The change in nanovesicles size due to stirring rate can also be explained by the intensification of micro mixing between the two phases, organic and aqueous during preparation that produces small droplets and hence small size vesicles [17].

Increasing stirring rate from 500 to $1200 \mathrm{rpm}$ showed a decrease of entrapment efficiency from 81.45 to $67.97 \%(p<0.05)$. This decrease entrapment efficiency upon increasing the stirring rate may be due to the increased surface area of the nanovesicles that caused the drug molecules exposed to the surface leading more drug escape to the medium [18].

\section{Effect of lipids}

Different lipids were evaluated for entrapment efficiency and particle size properties in the preparation of drug loaded nanovesicles (table 1). Inclusion of $P C$ and cholesterol showed an increased in entrapment efficiency up to $93.07 \%$. Nanoparticles prepared from lecithin, stearic acid and cholesterol exhibited lower entrapment (45.43\%) as compared to nanovesicles containing cholesterol, stearic acid and PC $(93.07 \%)$. Nanoparticles prepared from lipids of stearic acid and oleic acid in the presence of tween 80 exhibited the lowest entrapment efficiency of $37.41 \%$. It was evident from this that the inclusion of different types of lipids produced a significant effect on the entrapment efficiency $(p<0.001)$. Entrapment of $A m B$ increased from 77.63 to $93.07 \%$ upon adding stearic acid $(\mathrm{p}<0.05)$. A similar study showed liposomes prepared with stearic acid had better entrapment efficiency, and stearic acid acted like cholesterol in stabilizing liposomal structure [19]. The increase in entrapment of AmB could also be due to the amine group of the drug interacted with the carboxylic headgroup of stearic acid through establishment of electrostatic interactions [20]. However, the average particle sizes of vesicles with and without stearic acid was 192.5 and $193.3 \mathrm{~nm}$, respectively, showing that stearic acid didn't cause a significant change in particle size of the vesicles. The effect of use of different lipids didn't generally exhibit statistically significant change on particles size distribution of nanovesicles produced $(p=0.081)$.

\section{Effect of drug-lipid proportion}

The effect of drug-lipid proportion in nanovesicles preparation was assessed and presented in table 1 . The results indicated increasing the proportion of $\mathrm{AmB}$ significantly affected the particle size and entrapment efficiency. The particle size is decreased from 202.6 to $169.7 \mathrm{~nm}(\mathrm{p}<0.05)$ with a decrease in the drug's proportional from 1:5 to $1: 20$. The decrease in particle size upon decreased initial loading of $\mathrm{AmB}$ could be due to the decrease in the viscosity of the organic phase with resultant higher diffusion of the lipid solution as small droplets into aqueous medium leading in lower particle size of nanovesicles. A similar study showed increasing initial loading of AmB increased the particle size with widened size distribution [21]. Decreasing the proportion of drug from 1:5 to 1:20 resulted in a significant increase in DEE from 61.26 to $88.54 \%(\mathrm{p}<0.001)$. This shows decreasing the drugto-lipid ratio significantly enhanced the retention of the drug in the lipid. The increase in percent entrapment might be due to the presence of more internal phase in the lipid bilayer for dissolving the drug when lipid content is high [22]. The study also indicated the entrapment of $\mathrm{AmB}$ and stability of vesicles was found to be higher when the preparation temperature was about $75{ }^{\circ} \mathrm{C}$ as compared to lower temperatures such as $60^{\circ} \mathrm{C}$.

\section{Effect of ethanol-water composition}

The effect of different proportion of ethanol and water (PBS solution) in the process of vesicular preparations was investigated and the result is presented in table 1. At 2:1 ratio (66\% ethanol v/v in PBS), unstable preparation with sedimentation of drug at the bottom of the container was observed within hours, and those preparations were not further characterized. 1:1 (50\%) and 1:4 $(20 \%$ ethanol $\mathrm{v} / \mathrm{v})$ ratios showed changes in entrapment efficiency of the drug in nanovesicles. An increase in ethanol percentage from 20 to $50 \%$ caused the entrapment significantly decreased from 79.43 to $38.4 \%(p<0.01)$. However, the effect of an increase of ethanol percentage from 20 to $50 \%$ on nanovesicles diameter wasn't statistically significant $(p=0.09)$.

In general, factors evaluation study was conducted to evaluate the effects of factors; lipids composition, drug lipid proportion, ethanolwater composition and stirring rate on particle size and entrapment efficiency. The study demonstrated that, although particle size of vesicles were significantly affected by some of the factors, the vesicle sizes were still within the recommended rang of nanoparticles size from 100-250 $\mathrm{nm}$ for systemic drug delivery under all used ranges of the factors. However, drug entrapment efficiency was affected highly that ranges from 37.42 to $93.07 \%$ at varying compositions of the factors. All the factors also showed significant effects on drug entrapment efficiency $(\mathrm{p}<0.05)$, and the entrapment efficiency in some of the nanovesicles was very low. Thus, an optimal composition of factors was selected based on that to produce adequate entrapment efficiency. Hence, PC-cholesterol-stearic acid 20:4:1, drug-lipid 1:8, ethanol-aqueous medium 1:4 and stirring rate $1000 \mathrm{rpm}$ were selected as optimal factors composition for optimal AmB-MTF nanovesicles formulation development in the optimized formulation study.

\section{AmB-MTF 1:1 nanovesicles formulation}

An optimal formulation of AmB-MTF nanovesicles was prepared at the chosen values: PC-cholesterol-stearic acid 20:4:1, drug-lipid 1:8, AmBMTF 1:1, ethanol-water 1:4, and stirring rate $1000 \mathrm{rpm}$. This optimal factors composition was chosen based on the factors evaluation study. The AmB and miltefosine proportion of AmB: MTF 1:1 (w/w) was choosen based on their therapeutic doses to patients, and preliminary $\mathrm{AmB}$ and miltefosine interaction study on promastigotes (data not shown here).

AmB-MTF 1:1 nanovesicles showed vesicle size $145.6 \mathrm{~nm}$, poly dispersity index (PDI) 0.19 , zeta potential-27.3 $\mathrm{mV}$ and DEE $87 \%$ (table 2). PDI is used to describe the degree of non-uniformity of a size distribution of particles. According to international organization for standards (ISO), PDI values closer to 0 are seen with highly monodisperse standards, whereas PDI values 0.7-1.0 indicate that the sample has a very broad particle size distribution. In drug delivery applications using lipid-based carriers, a PDI of 0.3 and below is considered to be acceptable [23]. This may indicate the size distribution of AmB-MTF 1:1 nanovesicles (PDI $=0.19$ ) is within the acceptable range. The relative smaller particle size and PDI values in AmB-MTF 1:1 nanovesicles can be due to the surfactant nature of miltefosine that could produce smaller and uniform sized droplets and hence smaller nanovesicles during preparation [24]. Zeta potential results of AmB-MTF nanovesicles was- $27.3 \mathrm{mV}$, and $\mathrm{AmB}$ entrapment was $87.0 \%$. Particle size and zeta potential distributions of AmB-MTF 1:1 nanovesicles are presented in fig. 1.

\section{In vitro drug release study}

The results of in vitro drug release study of the AmB-MTF 1:1 nanovesicles formulation showed drug release of $99.51 \%$ in $72 \mathrm{~h}$. It is also evident from the result that about $50 \%$ of $\mathrm{AmB}$ was released in the first $6 \mathrm{~h}$, and about $93 \%$ of $\mathrm{AmB}$ was released in the first $24 \mathrm{~h}$ while the remaining undergo a more extended release up to a period of $72 \mathrm{~h}$ (fig. 2). The higher drug release rate of the poorly-water soluble AmB in the first $12 \mathrm{~h}$ may be due to the surfactant effect of miltefosine [25]. In vitro drug release study is performed in order to assess the potential of nanovesicles to control the release of drugs incorporated for prolonging the action [26].

Table 2: Particle size, PDI, Zeta potential and DEE evaluations of AmB-MTF nanovesicle

\begin{tabular}{lllll}
\hline Formulation & Particle size (nm) & PDI & Zeta potential (mV) & DEE (\%) \\
\hline AmB-MTF 1:1 nanovesicles & $145.6 \pm 8.4$ & $0.19 \pm 0.04$ & $-27.3 \pm 2.8$ & $87.0 \pm 4.2$ \\
\hline
\end{tabular}

Values represent mean \pm SD $(n=3)$ 


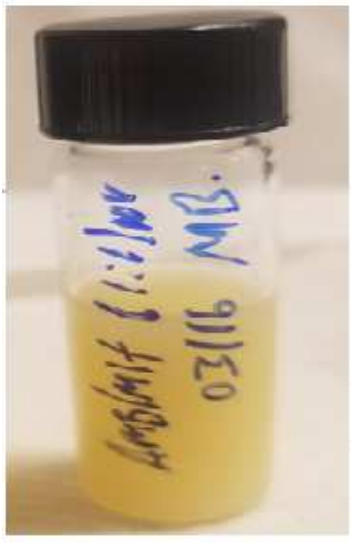

AmB/MTF 1:1 nanovesicle
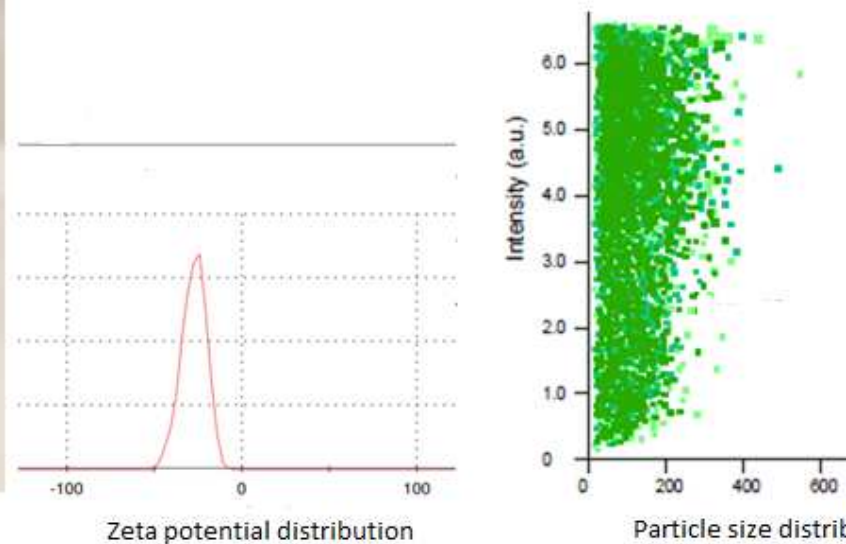

Particle size distribution

Fig. 1: Particle size (nm) and zeta potential distributions (mV) of the AmB-MTF 1:1 nanovesicles

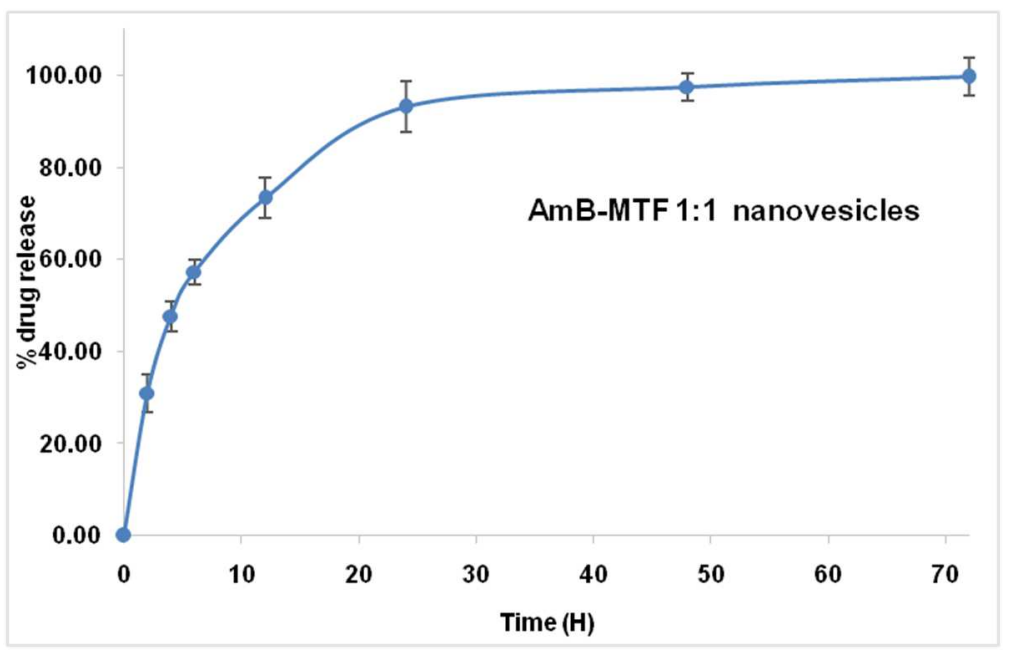

Fig. 2: In vitro drug release profile of AmB-MTF 1:1 nanovesicles in PBS (pH 7.4)

\section{Drug-excipients interaction study}

Drug-excipients interaction was studied using Fourier transform infrared (FTIR) spectroscopy, and the results are presented in fig. 3 . AmB demonstrates characteristic peaks in FTIR at $2920 \mathrm{~cm}^{-1}$ (due to its $\mathrm{CH}_{2}$ and $\mathrm{CH}_{3}$ stretching) and $3400 \mathrm{~cm}^{-1}$ (due to $\mathrm{OH}$ stretching) [27]. In this study, both plain AmB and AmB-MTF 1:1 nanovesicles showed these characteristic peaks of AmB at about the same wavelengths. Similarly, miltefosine exhibits its characteristic FTIR peaks at 2900 $\mathrm{cm}^{-1}\left(\mathrm{CH}_{2}\right.$ stretching) and $1500 \mathrm{~cm}^{-1}\left(\mathrm{CH}_{2}\right.$ bending) [28]. The results of this study also illustrated plain AmB and miltefosine, and AmB-MTF 1:1 nanovesicles showed these characteristic peaks at about the same wavelength. According to this study, there was no apparent interaction between the drugs and formulation excipients $[29,30]$.

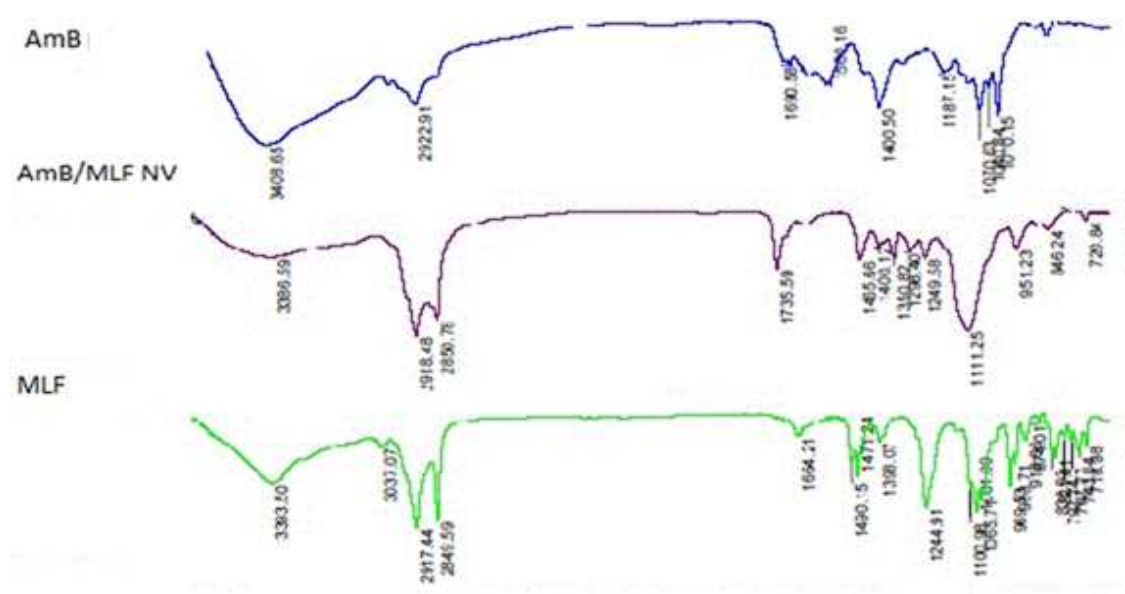

Fig. 3: FTIR spectra of AmB, miltefosine and AmB-MTF nanovesicles 


\section{CONCLUSION}

AmB-MTF nanovesicles were prepared by the ethanol injection method. The study showed that entrapment efficiency was significantly affected $(\mathrm{p}<0.01)$ by the effects of lipids, drug-lipid proportion, ethanol-water composition and stirring rate. Particle size of the nanovesicles were significantly affected $(\mathrm{p}<0.05)$ by druglipid proportion and stirring rate. The study also demonstrated that although both entrapment efficiency and particle size showed significant changes, drug entrapment efficiency was the most important response variable. Hence, an optimal composition of factors: PC-cholesterol-stearic acid 20:4:1, drug-lipid 1:8, ethanolaqueous medium 1:4 and stirring rate $1000 \mathrm{rpm}$ was chosen based on that to produce adequate entrapment efficiency for optimal AmBMTF nanovesicles preparation. Hence, optimized AmB-MTF1:1 nanovesicles was prepared at the chosen optimal factors composition, and its evaluation showed development of a successful formulation with very good compatibility, extended drug release, convenient particle size and high drug entrapment efficiency. This study concludes AmB-MTF 1:1 nanovesicles could be a safe and reliable therapeutic option over conventional AmB-MTF combination therapy. However, for the AmB-MTF 1:1 nanovesicles formulation to be used as better option of the conventional combinations in the treatment of visceral leishmaniasis, further antileishmanial investigations need to be conducted in vitro and in vivo.

\section{FUNDING}

The authors of this study would like to acknowledge the University of Gondar, Andhra University and the Ohio state university for their financial and material support.

\section{AUTHORS CONTRIBUTIONS}

All the authors listed in this manuscript have contributed equally.

\section{CONFLICT OF INTERESTS}

The author declares that there is no conflict of interest related to this report

\section{REFERENCES}

1. Musa A, Khalil E, Hailu A. Sodium stibogluconate-paromomycin combination for visceral leishmaniasis in East Africa randomized controlled trial. PLoS Negl Trop Dis 2012;6:1674.

2. WHO. Leishmaniasis. Available from: https://www.who.int/ news-room/fact-sheets/detail/leishmaniasis. [Last accessed on 12 Mar 2019].

3. Date AA, Joshi MS, Patravale VB. Parasitic diseases: liposomes and polymeric nanoparticles versus lipid nanoparticles. Adv Drug Delivery Rev 2007;59:505-21.

4. Wagner V, Minguez Menendez A, Pena J, Fernandez Prada C. Innovative solutions for the control of leishmaniases: nanoscale drug delivery. Curr Pharm Design 2019;25:1582-92.

5. Purkait B, Kumr A, Nandi N, Sardar A, Das S, Kumar S, et al. Mechanism of amphotericin $B$ resistance in clinical isolates of leishmanial donovani. Antimicrob Agents Chemother 2012;56:1031-41.

6. Shaw CD, Carter KC. Drug delivery: lessons to be learnt from Leishmania studies. Nanomedicine 2014;9:1531-44.

7. Tanga J, Srinivasanb S, Yuana W, Minga R. Development of a flow-through USP 4 apparatus drug release assay for the evaluation of amphotericin B liposome. Eur J Pharm Biopharm 2019;134:107-16.

8. Lankalapalli S, Tenneti VS, Nimmali SK. Design and development of vancomycin liposomes. Indian J Pharm Edu Res 2015;49:208-15.

9. Bose P, Kumar P, Dwivedi M. Hemoglobin guided nanocarrier for specific delivery of amphotericin B to Leishmania infected macrophage. Acta Trop 2016;15:148-59.

10. Momeni A, Rasoolian M, Momeni A, Navaei A. Development of liposomes loaded with antileishmanial drugs for the treatment of cutaneous leishmaniasis. J Liposome Res 2013;23:134-44.
11. AL-Quadeib BT, Radwan MA, Siller L, Horrocks B, Wrightd MC. Stealth amphotericin B nanoparticles for oral drug delivery: $I n$ vitro optimization. Saudi Pharm J 2015;23:290-302.

12. Wan S, Zhang L, Quan Y, Wei K. Resveratrol-loaded PLGA nanoparticles: enhanced stability, solubility and bioactivity of resveratrol for non-alcoholic fatty liver disease therapy. Royal Soc Open Sci 2018;5:18145.

13. Batzri S, Korn D. Single bilayer liposomes prepared without sonication. BBA-Biomembranes 1973;298:1015-9.

14. Ong S, Ming L, Lee K, Yuen K. Influence of the encapsulation efficiency and size of liposome on the oral bioavailability of griseofulvin-loaded liposomes. Pharmaceutics 2016;8:25.

15. Yang K, Delaney JT, Schubert US, Fahr A. Fast high-throughput screening of temoporfin-loaded liposomal formulations prepared by ethanol injection method. J Liposome Res 2012;22:31-41.

16. El-Say KM, Ahmed OA, Mohamed AI, Safo MK, Omar AM. El-Say $\mathrm{KM}$, et al. Zein alpha lipoic acid-loaded nanoparticles to enhance the oral bioavailability of dapoxetine: optimization and clinical pharmacokinetic evaluation. Int J Nanomed 2019;14:7461-73.

17. Shariare MH, Blagden N, De Matas M, Leusen F. The impact of formulation attributes and process parameters on black seed oil loaded liposomes and their performance in animal models of analgesia. Saudi Pharm J 2012;25:404-12.

18. Venkata NJ, Prasanna PM, Sakarkar SN, Prabha KS, Ramaiah PS. Microencapsulation techniques, factors influencing encapsulation efficiency. J Microencapsulation 2010;27:187-97.

19. Hsieh YF, Chen TL, Wang YT, Chang JH, Chang HM. Properties of liposomes prepared with various lipids. J Food Sci 2002;67:2808-13.

20. Cengiz A, Kahyaoglu T, Schröenb C, Berton Carabin C. Oxidative stability of emulsions fortified with iron: the role of liposomal phospholipids. J Sci Food Agric 2019;99:2957-65.

21. Italia JL, Sharp A, Carter KC, Warn P, Kumar R. Peroral amphotericin $\mathrm{b}$ polymer nanoparticles lead to comparable or superior in vivo antifungal activity to that of intravenous Ambisome ${ }^{\circledR}$ or Fungizone $\mathrm{T}^{\mathrm{TM}}$. Plosone 2011;6:1-11.

22. Kaur R, Sharma N, Tikoo K, Sinha VR. Development of mirtazapine loaded solid lipid nanoparticles for topical delivery: optimization, characterization and cytotoxicity evaluation. Int J Pharm 2020;586:119439.

23. Danaei M, Dehghankhold M, Hasanzadeh AF, Javanmard DR, Mozafari MR. Impact of particle size and polydispersity index on the clinical applications of lipidic nanocarrier systems. Pharmaceutics 2018;10:57.

24. Alonso L, Jeferson E, Cardoso S. Interactions of miltefosine with erythrocyte membrane proteins compared to those of ionic surfactants. Colloidal Surfaces B 2019;180:23-30.

25. Efentakis M, Al-Hmoud H, Buckton G, Rajan Z. The influence of surfactants on drug release from a hydrophobic matrix. Int J Pharm 1991;70:153-8.

26. Alvarez Berrios M, Aponte Reyes L, Diaz Figueroa L, Vivero Escoto J, Johnston A, Sanchez Rodriguez D. Preparation and in vitro evaluation of alginate microparticles containing amphotericin $\mathrm{b}$ for the treatment of candida infections. Int J Biomater 2020. https://doi.org/10.1155/2020/2514387.

27. D'Souza S. A review of in vitro drug release test methods for nano-sized dosage forms. Adv Pharm 2014:1-12. https://doi.org/10.1155/2014/304757.

28. Dorlo T, Eggelte T, Vriesa P, Beijnen J. Characterization and identification of suspected counterfeit miltefosine capsules. Analyst 2012;137:1265-74.

29. Kunasekaran V, Krishnamoorthy K. Compatibility studies of rasagiline mesylate with selected excipients for an effective solid lipid nanoparticles formulation. Int J Pharm Pharm Sci 2015;7:73-80.

30. Dawood NM, Abdal-Hammid SN, Hussien AA. Formulation and characterization of lafutidine nanosuspension for oral drug delivery system. Int J Appl Pharm 2018;10:10-30. 